\title{
SOME $p$-ADIC VERSIONS OF POLYA'S THEOREM ON INTEGER VALUED ANALYTIC FUNCTIONS
}

\author{
D. L. HILLIKER ${ }^{1}$ AND E. G. STRAUS ${ }^{2}$
}

ABstract. It is shown that functions whose values at the positive integers lie in a fixed algebraic number field with exponential growth restrictions on the conjugates and common denominators of these values must be polynomials if they can be represented by functions which are analytic in sufficiently large disks of $p$-adic planes.

1. Introduction. Polya's theorem characterizing the integer valued polynomials among the integer valued entire functions in terms of their growth rate has several distinct methods of proof and no doubt several distinct generalizations to analytic functions of a nonArchimedian variable. In this note we intend to consider functions whose values at the positive integers lie in a fixed algebraic number field $K$. Thus for any prime $p$ we can represent these functions as given at points of the $p$-adic unit disk with $p$-adic values determined by an embedding of $K$ in the $p$-adic plane $\Omega_{p}$ (that is the completion of the algebraic closure of $Q_{p}$ ). Of course, since the integers are no longer discrete the function is uniquely determined by these values.

We can represent the function by the formal interpolation series

$$
f(x)=\sum_{n=0}^{\infty} c_{n}(x-1)(x-2) \cdots(x-n)
$$

where

$$
c_{n}=\left(\Delta_{n} \Delta_{n-1} \cdots \Delta_{1} f\right)(n+1), \quad \Delta_{i} g(x)=\frac{g(x)-g(i)}{x-i} .
$$

Since all the terms beyond the first $n$ vanish for $x=n$ this series converges to $f(n)$ for positive integral values of $x$. From (1.2) we see that the values $c_{n}$ lie in $K$ and can be interpreted as lying in $\Omega_{p}$. If for some $R_{p} \geqq 1$ there exists a positive constant $M_{p}$ so that

Received by the editors February 5, 1970.

A MS 1969 subject classifications. Primary 1240; Secondary 3094.

Key words and phrases. Algebraic number theory, integer valued analytic functions, analytic functions, $p$-adic variable.

1 The research of the first author was sponsored in part by NSF Grant GP-8075.

2 The research of the second author was supported by NSF Grant GP-13164. 


$$
\left|c_{n}\right|_{p} \leqq M_{p} R_{p}^{-n}, \quad n=0,1,2, \cdots .
$$

Then the series (1.1) represents a function analytic in the disk $|x|_{p}<R_{p}$ of $\Omega_{p}$.

In $\$ 2$ we shall show conversely that generalized Taylor series of type (1.1) exist for functions analytic in a disk of $\Omega_{p}$ and in $\$ 3$ we show that if we combine suitable limitations on the heights (or rather "houses") of $f(n)$ and their denominators with suitable analyticity conditions on $f(x)$ in various $\Omega_{p}$ then we get criteria that characterize $f(x)$ as a polynomial.

In $\$ 4$ we consider various generalizations of the theorem in $\S 3$ both as concerns the set of points at which the values are assumed algebraic and the possibilities of relaxing the condition that all values lie in a fixed number field.

2. Generalized Taylor series in $\Omega_{p}$.

2.1 Lemma. Let $f(x)$ be analytic for $x \in \Omega_{p},|x|_{p} \leqq R$, where $R$ is in the valuation group of $\Omega_{p}$ (that is $R$ is a rational power of $p$ ) and let $\left\{x_{1}, x_{2}, \cdots\right\}$ be a sequence satisfying $\left|x_{n}\right|_{p} \leqq R ; n=1,2, \cdots$. Then $f(x)$ has a generalized Taylor series expansion

$$
f(x)=\sum c_{n}\left(x-x_{1}\right)\left(x-x_{2}\right) \cdots\left(x-x_{n}\right) .
$$

Here

$$
\left|c_{n}\right|_{p} R^{n} \rightarrow 0 \quad \text { as } n \rightarrow \infty .
$$

Conversely, every $f$ satisfying (2.2) and (2.3) represents a function analytic in $|x|_{p} \leqq R$.

Note. This interpolation theorem is typically stronger than the analogous theorem for analytic functions of a complex variable, where the points $x_{n}$ would have to be bounded away from the boundary of the disk of analyticity. A residual complication would remain if we were to deal with a radius $R$ which is not in the valuation group. In that case the rate of convergence of $\max _{i \leq n}\left|x_{i}\right|_{p}$ to $R$ would have to be restricted in a manner which depends on the func$\operatorname{tion} f$.

Proof. By a function analytic in $|x|_{p} \leqq R$, where $R$ is in the valuation group of $\Omega_{p}$, we mean a function

$$
f(x)=\sum_{n=0}^{\infty} a_{n} x^{n}
$$

with $\left|a_{n}\right|_{p} R^{n} \rightarrow 0$ as $n \rightarrow \infty$.

Now if the $x_{n}$ are all distinct then the $c_{n}$ can be computed by 


$$
c_{n}=\left(\Delta_{n} \Delta_{n-1} \cdots \Delta_{1} f\right)\left(x_{n+1}\right) \quad \text { where } \Delta_{i} g(x)=\frac{g(x)-g\left(x_{i}\right)}{x-x_{i}}
$$

Thus

$$
c_{n}=\sum_{k=0}^{\infty} a_{n+k} P_{n, k}\left(x_{1}, x_{2}, \cdots, x_{n+1}\right)
$$

where $P_{n, k}$ is a homogeneous polynomial of degree $k$ with integral coefficients. Taking absolute values on both sides of (2.6) we therefore get

$$
\left|c_{n}\right|_{p} R^{n} \leqq \max _{k \geqq 0}\left|a_{n+k}\right|_{p} R^{n+k} \rightarrow 0
$$

as $n \rightarrow \infty$. The case in which the $x_{n}$ are not all distinct can be handled by using derivatives in addition to difference quotients or as a limiting case of distinct $x_{n}$ since (2.6) shows that the $c_{n}$ are continuous functions of the $x_{n}$.

Now according to (2.7) the series (2.2) converges uniformly in $|x|_{p} \leqq R$ and thus represents an analytic function $g(x)$ in $|x|_{p} \leqq R$. The function $f(x)-g(x)$ is therefore analytic in $|x|_{p} \leqq R$ and has the infinite sequence $\left\{x_{1}, x_{2}, \ldots\right\}$ of zeros (counting multiplicities) in that disk. This is possible only if $f=g$.

\section{Main theorem.}

3.1 THEOREM. Let $K$ be an algebraic number field of degree $d$. Let $f(n) \in K$ for $n=1,2, \ldots$ and let $D_{n}$ be a positive integer so that $D_{n} f(i)$ is an integer of $K$ for $i=1,2, \cdots, n$.

Assume

$$
\limsup _{n \rightarrow \infty} \frac{\log \left(\max \left\{D_{n}, \sqrt{f(n) \mid}\right\}\right)}{n}=\gamma<\infty
$$

where, as usual, $\overline{|\alpha|}$ stands for the maximal absolute value of the conjugates of $\alpha$.

Let $P$ be a finite set of primes such that for each $p \in P$ the field $K$ has $e_{p}$ isometric (conjugate) embeddings in $\Omega_{p}$ for which $f(n)$ are the values of a function analytic in a disk $|x|_{p} \leqq R_{p}$ where $R_{p} \geqq 1$.

$$
\text { If }
$$

$$
\prod_{p \in P}\left(p^{1 /(p-1)} R_{p}\right)^{\operatorname{eop}}>2^{d} e^{2 d \gamma} .
$$

then $f(x)$ is a polynomial. 
Note that (3.3) is satisfied for any $d$ and $\gamma$ if $f$ is entire in any $\Omega_{p}$. In that case we could combine growth conditions on $\max |f(x)|_{p}$ with conditions on $f(n)$ that are somewhat weaker than those in (3.2).

Proof. Since (3.3) is a strict inequality we may assume that the $R_{p}$ in question lie in the valuation group of $\Omega_{p}$. Thus for each of the given embeddings of $K$ in $\Omega_{p}$ we get expansions of $f(x)$ in the form (1.1) and according to (2.7) we have constants $M_{p}$ so that

$$
\left|c_{n}\right|_{p} \leqq M_{p} R_{p}^{-n} \text { for } p \in P \text {. }
$$

Also from (2.5) we get

$$
c_{n}=\sum_{i=1}^{n+1}(-1)^{n-i} \frac{f(i)}{(i-1) !(n-i) !} .
$$

Thus, setting $c_{n}^{\prime}=(n-1) ! c_{n}$ we get

$$
D_{n} c_{n}^{\prime}=\sum_{i=1}^{n+1}\left(\begin{array}{l}
n-1 \\
i-1
\end{array}\right)(-1)^{n-i} D_{n} f(i)
$$

where the right side is an integer of $K$. Using (3.2) we get

$$
\overline{\left|D_{n} c_{n}^{\prime}\right|}<c 2^{n} e^{2 n(\gamma+\epsilon)}
$$

or, for the norm $N\left(D_{n} c_{n}^{\prime}\right)$, we have

$$
\left|N\left(D_{n} c_{n}^{\prime}\right)\right| \leqq \longdiv { D _ { n } c _ { n } ^ { \prime } | ^ { d } } < c 2 ^ { n d } e ^ { 2 n d ( \gamma + \epsilon ) }
$$

for all large $n$.

On the other hand we get from (3.4), and the fact that all conjugates of $D_{n} c_{n}^{\prime}$ have $p$-adic absolute value $\leqq 1$, that

$$
\begin{aligned}
\left|N\left(D_{n} c_{n}^{\prime}\right)\right|_{p} & \leqq\left|D_{n}(n-1) ! c_{n}\right|_{p}^{e_{p}} \leqq\left|(n-1) ! c_{n}\right|_{p}^{e_{p}} \\
& \leqq\left|p^{-n /(p-1)+{ }^{\epsilon} n} M_{p} R_{p}^{-n}\right|^{0} p \\
& <\left(p^{-1 /(p-1)} R_{p}^{-1}+\epsilon\right)^{n e_{p}}
\end{aligned}
$$

for all large $n$.

Now, if $c_{n} \neq 0$, then since $D_{n} c_{n}^{\prime}$ is an integer we must have

$$
\left|N\left(D_{n} c_{n}^{\prime}\right)\right| \geqq \prod_{p \in P}\left|N\left(D_{n} c_{n}^{\prime}\right)\right|_{p}^{-1}
$$

Substituting (3.8) and (3.9) in (3.10) we get

$$
\left(2^{d} e^{2 d \gamma}+\epsilon\right)^{n}>\prod_{p \in P}\left(p^{1 /(p-1)} R_{p}-\epsilon\right)^{n e_{p}}
$$


for all large $n$. Since (3.11) contradicts (3.3) we must have $c_{n}=0$ for all large $n$; in other words $f(x)$ is a polynomial.

We may ask to what extent condition (3.2) is necessary for Theorem 3.1. The following example shows how vital it is.

3.12 EXAMPLE. Let $\phi(n)$ be any monotonically increasing function which tends to $\infty$. Then the function

$$
f(x)=\sum_{n=0}^{\infty} \prod_{k=1}^{\infty} p_{k}^{\left[n \phi(n) /\left(2^{k} \log p_{k}\right)\right]} \frac{(x-1)(x-2) \cdots(x-n)}{n !}
$$

has integral values at the integers with

$$
\log |f(n)| \leqq \log \left(2^{n} e^{n \phi(n)}\right)=n(\phi(n)+\log 2)
$$

while there exist positive constants $k_{p}$ with

$$
\left|c_{n}\right|_{p}^{1 / n}<p^{-k_{p} \phi(n)} \rightarrow 0 \text { as } n \rightarrow \infty
$$

so that $f(x)$ is $p$-adically entire for all $p$.

4. Generalizations. Instead of considering the values $f(n)$ we could have considered other $p$-adically bounded sequences $\left\{x_{1}, x_{2}, \ldots\right\}$ of algebraic numbers; the main complication would arise in assessing the algebraic behavior of $c_{n}$. The analog of (3.5) is

$$
c_{n}=\sum_{i=1}^{n+1} f\left(x_{i}\right) \prod_{j=1 ; j \neq i}^{n}\left(x_{i}-x_{j}\right)^{-1}
$$

so that we need bounds on the denominators and the absolute values introduced by the factors $\prod\left(x_{i}-x_{j}\right)^{-1}$ on the right. In the case of the integers this task was simplified by the use of the common denominator $(n-1)$ ! and the binomial coefficients.

Instead of considering values in a fixed algebraic number field $K$ we could let the fields $K_{n}$ generated by $f\left(x_{1}\right), \cdots, f\left(x_{n}\right)$ grow in degree with $n$. The corresponding estimates of the norms of the coefficients $c_{n}$ would then also involve varying exponents $d_{n}$.

Finally, as mentioned, we could strengthen the analyticity conditions on the $p$-adic functions $f(x)$ and correspondingly weaken the arithmetical conditions on $f(n)$.

We did not explicitly emphasize the possible interpretation of $f(x)$ as a complex analytic function. However, for example our condition (3.2) would be an immediate consequence of the hypothesis that $f(x)$ is an integral valued entire function of exponential type $\leqq \gamma$. 


\section{REFERENCES}

1. D. L. Hilliker, On analytic functions which have algebraic values at a convergent sequence of points, Trans. Amer. Math. Soc. 126 (1967), 534-550. MR 34 \#4207.

2. - Algebraically dependent functions of a complex and p-adic variable, Proc. Amer. Math. Soc. 19 (1968), 1052-1056. MR 38 \#135.

3. G. Pólya, Über ganzwertige ganze Funktionen, Rend Circ. Mat. Palermo 40 (1915), 1-16.

Pennsyluania State University, University Park, Pennsylvania 16802

University of California, Los Angeles, California 90024 